\title{
Mehr als kalter Kaffee?
}

\section{Seit Ende letzten Jahres liegt eine vorläufige Version eines Verhaltenskodexes für die Kaffeewirtschaft vor. Es soll die ruinösen Bedingungen für Kaffeeprodu- zenten verbessern. Das Abkommen ist ein großer Schritt in die richtige Rich- fung, doch noch weit von seinem Ziel entfernt.}

$\mathrm{K}$ Von Gertrud Falk affee ist weltweit nach Erdöl das meist gehandelte Produkt. Der Lebensunterhalt von etwa 25 Millionen Menschen in tropischen und subtropischen Ländern ist direkt abhängig von der Kaffeeproduktion. Seit Ende der 90er Jahre befindet sich der Kaffeemarkt in einer Preiskrise, die durch Überproduktion verursacht wurde und verheerende Folgen für die Kaffeebauern hat. Seit Mai 2003 erarbeiten auf Initiative des Deutschen Kaffeeverbands (DKV) und der Gesellschaft für Technische Zusammenarbeit (GTZ) VertreterInnen von Produzentenverbänden, europäischen Kaffeehändlern und -röstern sowie zivilgesellschaftliche Organisationen in einem partizipativen Prozess den „Common Code for the Coffee Community“ (CCCC). Mit diesem Verhaltenskodex soll durch Qualitätsverbesserung von Produktion und Produkt eine nachhaltigere Produktionsweise in der Kaffeewirtschaft erreicht werden, die zu einem höheren Einkommen der Kaffeebauern, dem Schutz der Umwelt und der Verbesserung der Qualität des Kaffees führen soll. Eine erste, noch unvollständige Version des CCCC wurde am zehnten September 2004 der Presse vorgestellt.

\section{Scheitern des Kaffeeabkommens}

Seit dem Scheitern des marktregulierenden Kaffeeabkommens im Jahr 1989 wurde die Produktion von Kaffee weltweit über die Nachfrage hinaus gesteigert. Viele Kaffee produzierenden Länder weiteten ihre Produktion in der Hoffnung auf hohe Deviseneinnahmen aus. Gleichzeitig erlaubten verbesserte Verarbeitungsmethoden die Beimischung eines erhöhten Anteils der bitteren Robusta-Bohnen, die nicht so hohe Anforderungen an die Boden- und Klimabeschaffenheit stellen wie die hochwertigeren Arabica-Pflanzen.

Allen voran entwickelte sich Vietnam innerhalb von zehn Jahren zum weltweit zweitgrößten Kaffeeproduzenten nach Brasilien. Gefördert von der Weltbank trug dieses „Entwicklungsprojekt“ zur
Verarmung von unzähligen Kaffeebauern und ihren Familien bei. Denn der Kaffeemarkt ist relativ unelastisch. Niedrige Preise führen kaum zu höherem Konsum. So sank der Anteil der Produzenten am Konsumpreis laut kolumbianischem Kaffeeverband von 30 auf weniger als acht Prozent. Die Entwicklungsorganisation Oxfam nahm diese Situation zum Anlass ihrer Kaffeekampagne und griff die Kaffeeindustrie an, deren Gewinne während der Preiskrise anstiegen.

\section{- Kaffeeindustrie unter Handlungszwang}

Angesichts des öffentlichen Druckes durch die Oxfam-Kampagne und des Absinkens der Qualität des Rohkaffees ergriff der Deutsche Kaffeeverband im Jahr 2002 die Initiative zu einem Public-PrivatePartnership-Projekt (PPP) mit der GTZ. Dessen Ziel war die Erarbeitung und Implementierung eines Verhaltenskodex für die Kaffeewirtschaft in einem kooperativen Prozess verschiedener Interessenvertreter. Dabei wurde von vornherein der gewöhnliche Kaffee anvisiert, der weder zertifiziert ist noch als Spezialitätenkaffee verkauft wird und etwa 95 Prozent des Marktes ausmacht.

Im Mai 2003 wurde das Steuerungsgremium des Prozesses gegründet. Es setzt sich aus den drei konstituierenden Gruppen Produzenten, Industrie und Handel, Zivilgesellschaft sowie außerordentlichen Mitgliedern zusammen. Es trifft im

\section{Inserieren Sie in Ökologisches Wirtschaften!}

Sprechen Sie genau Ihre Zielgruppe an. Erscheint 5 x jührlich.

Fordern Sie noch heute die Mediadaten an!

Frau Dagmar Huber berät Sie gerne.

\section{Kontakt: Dagmar Huber}

Waltherstraße 29, 80337 München Fon 089/544 184 12, Fax 089/544 18449 E-Mail: huber@oekom.de
Konsens die Entscheidungen über Verfahren und Ergebnisse. In der Produzentenkammer sind Organisationen aus Kolumbien, Brasilien, Guatemala, Elfenbeinküste, Angola, Indonesien, Indien, Vietnam und Ostafrika vertreten. Handel/Industrie werden durch Kraft Foods, Nestlé, Neumann Kaffee Gruppe, Volcafé, Sara Lee und Tchibo repräsentiert. In der Gruppe der Zivilgesellschaft arbeiten die International Union of Food Workers (IUF), FIAN (mit Mandat von VENRO), Oxfam, die Christliche Initiative Romero, Rainforest Alliance und bis kurz vor der Veröffentlichung des Kodexentwurfs auch Greenpeace mit. Außerordentliche Mitglieder der Steuerungsgruppe sind die Internationale Kaffeeorganisation (ICO), die Internationale Arbeitsorganisation (ILO), der niederländische Zertifizierer Utz Kapeh, die Weltbank, das International Institute for Sustainable Development (IISD) für die Sustainable Coffee Partnership sowie das Bundesministerium für wirtschaftliche Zusammenarbeit und Entwicklung und das Bundesministerium für Wirtschaft und Arbeit. Zur Erarbeitung der Inhalte des CCCC wurden drei Arbeitsgruppen entlang der Nachhaltigkeitsdimensionen Wirtschaft, Soziales und Umwelt eingesetzt, die ebenfalls tripartistisch besetzt waren. Die Idee war dabei, einen dynamischen Kodex zu erarbeiten, der den Beteiligten eine schrittweise Umsetzung der Standards ermöglicht. Unterstïtzt wurde die Arbeit von Steuerungsgremium und Arbeitsgruppen durch das bei der GTZ angesiedelte Projektsekretariat, das sich allerdings die Kritik der Industrienähe und teilweisen Intransparenz gefallen lassen musste.

Herausgekommen ist ein noch unvollständiger Kodex, der die Einhaltung zentraler internationale Menschenrechts- und Arbeitsnormen sowie das Verbot von hochgefährlichen Pestiziden zur Teilnahmebedingung macht und darauf aufbauend Verbesserungen der Produktionsstandards verlangt.

\section{Zentrale Konfliktthemen}

Zentraler Diskussionspunkt war die Forderung an die internationalen Händler, den ProduzentInnen höhere Preise für den Kaffee zu garantieren, der nach den CCCC-Standards produziert wird. Denn Ausgangspunkt des gesamten Prozesses war schließlich, dass Kaffeebauern mit dem Verkauf ihres Kaffees ihre Kosten nicht mehr decken konnten. Hier erwies sich jedoch das internationale Kartellrecht als Hindernis. Da die großen europäischen Kaffeehändler und -röster an einem Tisch saßen, durfte nichts besprochen werden, 
was unter den Verdacht von Preisabsprachen fallen konnte. Möglich war nur die Integration eines zwischen Produzenten und Händler frei verhandelbaren Preisaufschlags für die CCCC-Qualität. Im Laufe des Prozesses wurde immer deutlicher, dass der Kodex sich nicht gleichermaßen an die gesamte Angebotskette von Rohkaffee wandte, sondern vor allem den Produzenten Standards setzt. Dies führte zu der Forderung von Produzenten und Zivilgesellschaft, dass es zusätzlich einen so genannten „,buyer's code“ für Handel und Industrie geben müsse. Während diese das zunächst ablehnten und nur für alle Beteiligten geltende Richtlinien akzeptieren wollten, lenkten sie kurz vor der Medien-Präsentation des Kodexentwurfs ein. Allerdings existiert zu den Regeln für Industrie und Handel bisher nur ein Entwurf. Wesentliche Forderungen von Produzenten und Zivilgesellschaft wollen Industrie und Handel bisher nicht erfüllen:

- die Verpflichtung zum Kauf signifikanter Mengen des CCCC-Kaffees

- die Verpflichtung zum Kauf kontinuierlich steigender Mengen CCCC-Kaffees

- die nachträgliche Veröffentlichung des durchschnittlichen CCCC-Preisaufschlags per Land

Bis kurz vor dem Gang an die Öffentlichkeit waren wesentliche Inhalte des Kodex umkämpft. Uneinigkeit herrschte dabei auch unter den Industrieunternehmen. So waren sie gespalten in den Fragen nach der Unabhängigkeit des Verifizierungssystems und der Verwendung von genverändertem Saatgut, die vor allem von der Zivilgesellschaft gefordert wurden. Während die zivilgesellschaftlichen Organisationen die Unabhängigkeit der Verifizierung schließlich durchsetzen konnten, waren sie beim Thema Saatgut nur zum Teil erfolgreich. Das Steuerungsgremium einigte sich nur auf einen Minimalkonsens, der besagt, dass in den nächsten fünf Jahren unter dem Namen des CCCC kein genverändertes Saatgut verwendet und nicht mit genverändertem Kaffee gehandelt werden darf. Über die Ausdehnung auf die gesamte Unternehmenspolitik der beteiligten Firmen sowie über die Frage von Patenten soll nach einem ICO-Seminar zu diesem Thema im nächsten Jahr entschieden werden. Greenpeace ging dieser Entschluss nicht weit genug und trat daher am Abend vor der öffentlichen Vorstellung des CCCC aus dem Prozess aus.

\section{Chancen und Grenzen des CCCC}

Der CCCC wird die Kaffeekrise nicht lösen. Dazu wäre ein neues Kaffeeabkommen nötig. Die Über- produktion, zu der die beteiligten Unternehmen beigetragen haben, wird durch den Verhaltenskodex nicht gebremst. Ob er tatsächlich zur Verbesserung des Lebensstandards von Kaffeebauern, ihren Familien und PlantagenarbeiterInnen führen wird, wird vor allem davon abhängen, dass die an CCCC beteiligten Händler und Röster den Kaffeebauern genügend Anreize bieten, um die Standards des Kodex einzuhalten. Diese können zum Beispiel in Form von höheren Preisen, Abnahmegarantien oder auch qualifizierter Weiterbildung gegeben werden. Handels- und Röstunternehmen müssen dazu in der nun beginnenden Implementierungsphase Farbe bekennen.

Der CCCC fordert Kleinbauern auf sich zu organisieren, da die Herkunft des Kaffees vom Konsumenten bis zum Volumen von einem Container Rohkaffee zurückverfolgbar werden soll. Dies stellt für viele Kaffeebauern eine Herausforderung dar, denn der Organisationsgrad des Sektors ist gering. Durch bessere Kooperation, verknüpft mit dem angestrebten besseren Zugang zu Marktinformationen, können die Bauern so gegebenenfalls ihre Verhandlungsmacht gegenüber den Händlern stärken.

Bei großer Beteiligung am CCCC besteht langfristig auch die Möglichkeit, einen Preisaufschlag für CCCC-Qualität an den Börsen zu erzielen. Es müssten dann Wege gefunden werden, den Preisaufschlag an die Kaffeeproduzenten zurückzugeben. Für die Handels- und Röstunternehmen stellt der CCCC auch unabhängig von der Frage der Anreize für Kaffeebauern eine Herausforderung dar. So erfüllen nicht alle die Mindeststandards zur Teilnahm am Kodex, wie zum Beispiel die Gewährleistung von Gewerkschaftsfreiheit und Kollektivverhandlungen. Für viele ArbeitnehmerInnen der Kaffeebranche kann der CCCC so die Möglichkeit zur gewerkschaftlichen Organisation bieten.

Den Nationalökonomien der Produzentenländer stellt der Kodex keine bedeutenden neuen Einkommensquellen in Aussicht. Denn er verlangt keine stärkere Verlagerung der Wertschöpfungskette in die Produzentenländer. Dies wäre jedoch nötig, um die Länder langfristig aus der Abhängigkeit von Rohstoffexporten abzulösen.

Der CCCC macht deutlich, dass vielen Menschen geholfen wäre, wenn Unternehmen und Plantagenbetreiber sich an die bereits bestehenden internationalen Normen und nationalen Gesetze halten würden. Denn die Standards des Kodex sind nicht neu. Die striktere Kontrolle bereits bestehender Normen wäre vermutlich effektiver als Anreize zu ihrer Einhaltung mit einem freiwilligen
Kodex zu schaffen. Auch im Kaffeesektor besteht die Gefahr, dass sich Unternehmen mit Einzelmaßnahmen zur Wahrnehmung ihrer gesellschaftlichen Verantwortung die Weste weiß waschen und gleichzeitig Arbeitnehmerrechte verletzen und den Kaffeebauern Hungerpreise zahlen.

\section{Zukunft des Codes}

Die GTZ wird das Projekt weiterhin finanziert vom Bundesministerium für wirtschaftliche $\mathrm{Zu}$ sammenarbeit und Entwicklung (BMZ) begleiten. Der DKV hat die Zuständigkeit für die weitere Arbeit an die European Coffee Federation abgegeben. Als weiterer öffentlicher Financiers unterstïtzt das Schweizer Wirtschaftsministerium den Prozess in Zukunft.

Der CCCC soll organisatorisch an die ICO angegliedert werden. Bei der Vorstellung des CCCC auf der Jahresversammlung der ICO im September 2004 kritisierten jedoch viele Produzentenländer den CCCC, da er ihnen zu einseitig die Kosten der nachhaltigen Produktion aufbürde. Auch Oxfam kritisiert in einem offenen Brief, dass der Kodex den Produzenten zu wenig Anreize zur Teilnahme biete und die Industrie zu wenig in die Pflicht nehme.

Für die nun beginnende Implementierungsphase gibt es noch keine CCCC-eigene Pilotprojekte. Bisher wollen lediglich die beteiligten Handels- und Röstunternehmen versuchen, den Kodex in ihre bestehenden Projekte einzugliedern. Weiterhin führt die GTZ PPP-Projekte mit den Industrieunternehmen zum CCCC durch. Zivilgesellschaftliche Organisationen stehen bisher außen vor. Dadurch droht jedoch das tripartistische Steuerungsgremium die Kontrolle über die Einführung des Kodex zu verlieren.

\section{Anmerkung}

(1) Der Verhaltenskodex sowie weitere Informationen dazu sind auf der Internetseite www.sustainable-coffee.net abrufbar.

\section{Die Autorin}

Gertrud Falk arbeitet bei der Menschenrechtsorganisation FIAN zum CCCC.

Kontakt: FIAN, Overwegstraße 31, 44625 Herne. E-Mail: g.falk@fian.de 
(c) 20I0 Authors; licensee IÖW and oekom verlag. This is an article distributed under the terms of the Creative Commons Attribution Non-Commercial No Derivates License (http://creativecommons.org/licenses/by-nc-nd/3.o/), which permits unrestricted use, distribution, and reproduction in any medium, provided the original work is properly cited. 